Originalveröffentlichung in: Bernd U. Schipper (Hrsg.), Ägyptologie als Wissenschaft,

Adolf Erman (1854-1937) in seiner Zeit, Berlin und New York 2006, S. 224-247

\title{
Bruch und Aufbruch Adolf Erman und die Geschichte der Ägyptologie
} Wolfgang Schenkel

\section{Die Herausbildung der Ägyptologie als wissenschaftlicher Disziplin}

Bonaparte und Champollion bezeichnen den Beginn einer Entwicklung, die zur Etablierung der Ägyptologie als einer gesonderten wissenschaftlichen Domäne geführt haben: Napoléon Bonaparte, der auf seinem Kriegszug nach Ägypten 1798 eine wissenschaftliche Kommission mit an Bord nahm, die nicht zuletzt auch - in zuvor nie gekannter Intensität - den Spuren der pharaonischen Vergangenheit nachging; Jean François Champollion, dem bekanntlich der entscheidende Durchbruch zur Lesbarmachung der schriftlichen Überlieferung aus der pharaonischen Zeit gelang. Damit gab es aber noch keine Ägyptologie. Diese war als eine gesonderte wissenschaftliche Domäne erst um 1860 etabliert.

1849-1859 waren nach und nach die Bände des gewaltigen DenkmälerWerks von Richard Lepsius erschienen, die Ernte der letzten der großen wissenschaftlichen Expeditionen. Es lagen nach dessen Abschluss nahezu alle Denkmäler, die man in raschen Surveys gewinnen konnte, in zuverlässigen Publikationen bereit. 1851 war es Emmanuel de Rougé zum ersten Mal gelungen, einen zusammenhängenden hieroglyphischen vollständigen Text zu erklären. Nach einem weiteren Jahrzehnt war die Bearbeitung der Texte zum Alltagsgeschäft geworden. In die Museen waren große Mengen von Originalen eingebracht worden, so die Sammlungen Salt, Drovetti, Passalacqua, um nur einige zu nennen, in die Museen von Turin, Paris, London und Berlin. An Hochschulen waren die ersten Lehrstühle für Ägyptologie eingerichtet worden. Auf den für Champollion 1831 ad personam eingerichteten Lehrstuhl am Collège de France war 1860 zum ersten Mal ein Wissenschaftler berufen worden, den man ohne wenn und aber als Ägyptologen bezeichnen darf, Emmanuel de Rougé. Außerhalb Frankreichs war in Berlin Lepsius als Ägyptologe aufgebaut 
worden: durch Berufung zum Professor 1846, Wahl in die Akademie 1850, Bestimmung zum Leiter der ägyptischen Abteilung des Museums 1855. Daran anschließend wurden ab den 60er Jahren nach Pariser und Berliner Vorbild zunehmend mehr Lehrstühle gegründet. 1863 begründete Heinrich Brugsch die erste ägyptologische Fachzeitschrift, die Zeitschrift für ägyptische Sprach- und Alterthumskunde, wie der Titel zunächst lautete, die Ägyptische Zeitschrift oder kurz die $\ddot{A} Z$, wie sie noch nach der Mitte des 20. Jahrhunderts genannt wurde. Erinnert sei schließlich auch daran, dass, wie Stephen R. K. Glanville in seiner Cambrigder Antrittsvorlesung von 1947 feststellt, 1863 mit der Berufung August Mariettes durch den Khediven Ismail der erste Schritt zu einer Etablierung der Ägyptologie in Ägypten getan wurde und dass 1859 das Wort "egyptology" im New English Dictionary zum ersten Mal im Druck erscheint. ${ }^{1}$ (Was das erste Auftreten des Wortes angeht, ist daran sicherlich für die anglophone Welt eine Epochenschwelle markiert; im Französischen hat der Wortgebrauch jedoch einen etwas älteren Vorlauf. ${ }^{2}$ )

\section{Vom Sammeln zum Erschließen}

Unter dem Gesichtspunkt des methodischen Forschritts betrachtet, beginnt mit den 1860er Jahren keine neue Epoche. Im Grunde genommen dümpelt die nur nach außen hin als neue Disziplin sichtbare Ägyptologie weiter im Fahrwasser, in dem sie seit Bonaparte und Champollion geschwommen war: Es wird weiterhin Material gesammelt und es werden Teilauswertungen der angehäuften Materialien vorgenommen. Eine Neuorientierung bricht sich dagegen um 1880 Bahn. Um diese Zeit schreitet man vom Sammeln des sich Anbietenden zum systematischen Erschließen des gesamten Quellenfundus.

\section{Die Phase des Sammelns}

Beginnend mit der Expedition unter Bonaparte finden in Abständen von zwei Jahrzehnten Surveys in Ägypten statt. Deren Ergebnisse liegen jeweils etwa ein, zwei Jahrzehnte später abgeschlossen im Druck vor. Es erscheinen (s. Abb. 1): 


\begin{tabular}{|c|c|c|c|}
\hline Generation & I & II & III \\
\hline Jahrzehnt & $\begin{array}{c}\text { (Bonaparte } \\
* 1769) \\
(\text { Jomard } \\
* 1777)\end{array}$ & $\begin{array}{c}\text { Champollion } \\
* 1790 \\
\text { (Rosellini } \\
* 1800)\end{array}$ & $* 1810$ \\
\hline $1770-$ & & & \\
\hline $1780-$ & & & \\
\hline $1790-$ & & & \\
\hline $1800-$ & Expedition & & \\
\hline $1810-$ & & & \\
\hline $1820-$ & Description & Expedition & \\
\hline $1830-$ & & Monuments & Expedition \\
\hline $1840-$ & & & Denkmäler \\
\hline $1850-$ & & & \\
\hline $1860-$ & & & \\
\hline $1870-$ & & & \\
\hline $1880-$ & & & \\
\hline
\end{tabular}

Abb. 1: Sammeln / Denkmäler: Protagonisten aus drei Generationen bis zur Veröffentlichung

- die Ergebnisse der französischen Expedition unter Bonaparte aus den Jahren 1798 bis 1801, die maßgeblich durch Edmé François Jomard vorangetriebene Description de l'Égypte, in den Jahren 1809 bis 1826, ${ }^{3}$

- die Ergebnisse der französisch-toskanischen Expedition unter Champollion und Ippolito Rosellini aus den Jahren 1828 und 1829, die Monuments de l'Égypte et de la Nubie von Champollion, posthum in den Jahren 1835-1845 und die Monumenti dell'Egitto e della Nubia von Rosellini, in den Jahren $1832-1844 ;^{5}$

- die Ergebnisse der preußischen Expedition unter Lepsius aus den Jahren 1842-1845, die Denkmaeler aus Aegypten und Aethiopien von Lepsius, in den Jahren 1849-1859. ${ }^{6}$

Nicht Schritt halten mit der Gewinnung des Materials konnte die Auswertung. Immerhin spielten von der französischen Expedition gewonnene Materialien, namentlich die Rosettana, der "Stein von Rosette“, und die von einzelnen Antikeninteressenten aquirierten oder dokumentierten Objekte, namentlich ein Obelisk aus Philae und Zeichnungen aus Abu Simbel, eine Schlüsselrolle bei 
der Entzifferung der Hieroglyphen. Champollion dagegen kam infolge seines frühen Todes nicht einmal mehr zum Publizieren seiner Materialien, geschweige denn zur Ausschöpfung derselben. Nicht zuletzt blieb schlecht ausgewertet das immense und qualitätvollste Material, dasjenige, das Lepsius zusammengetragen hatte. Lepsius selbst, der noch Einzeluntersuchungen geleistet hatte, war zu sehr von den Tagesgeschäften in Beschlag genommen und trotz aller seiner ägyptischen Interessen doch immer noch mehr in der klassischen Antike zu Hause. Infolgedessen war er ägyptologisch zunehmend nicht mehr recht auf der Höhe der Zeit. Schließlich musste er in seinen späten Jahren „zur Sanierung seiner durch Spekulation zerrütteten Vermögensverhältnisse“ einen guten Teil seiner Arbeitskraft auf die Leitung der Königlichen Bibliothek in Berlin verwenden. ${ }^{\text {? }}$

Um zwei Jahrzehnte zeitversetzt gegenüber der Gewinnung der Denkmäler schreitet in Etappen die Erforschung der ägyptisch-sprachigen Textzeugnisse voran. Man kann hier drei Forschergenerationen unterscheiden (s. Abb. 2):

\begin{tabular}{|l|l|l|l|l|l|l|l|}
\hline $\begin{array}{l}\text { Gene- } \\
\text { ration }\end{array}$ & I & \multicolumn{3}{|l|}{ II } & II/III & III \\
\hline $\begin{array}{l}\text { Jahr- } \\
\text { zehnt }\end{array}$ & $\begin{array}{l}\text { Cham- } \\
\text { pollion } \\
* 1790\end{array}$ & $\begin{array}{l}\text { Lepsius } \\
* 1810\end{array}$ & $\begin{array}{l}\text { de Rougé } \\
* 1811\end{array}$ & $\begin{array}{l}\text { Goodwin } \\
* 1817\end{array}$ & $\begin{array}{l}\text { Chabas } \\
* 1817\end{array}$ & $\begin{array}{l}\text { Brugsch } \\
* 1827\end{array}$ & $\begin{array}{l}\text { Erman } \\
* 1854 \\
\text { (Petrie } \\
* 1853)\end{array}$ \\
\hline $1770-$ & & & & & & \\
\hline $1780-$ & & & & & & \\
\hline $1790-$ & & & & & & \\
\hline $1800-$ & & & & & & \\
\hline $1810-$ & & & & & & \\
\hline $1820-$ & & & & & & \\
\hline $1830-$ & & & & & & \\
\hline $1840-$ & & & & & & \\
\hline $1850-$ & & & & & & \\
\hline $1860-$ & & & & & & \\
\hline $1870-$ & & & & & & \\
\hline $1880-$ & & & & & & \\
\hline $1890-$ & & & & & & \\
\hline $1900-$ & & & & & & \\
\hline
\end{tabular}

Abb. 2: Sammeln / Sprache: Lebenszeit der Protagonisten aus drei Generationen 
- Champollion, geboren 1790, dem der Durchbruch bei der Entzifferung gelang und dem sich in rasantem Tempo das Pharaonisch-ägyptische in Grammatik und Wortschatz zu erschließen begann; ${ }^{8}$

- die Generation der zwischen 1810 und 1820 Geborenen, die die Entzifferung nach dem vorzeitigen Tod Champollions konsolidierten - Lepsius ${ }^{9}$ - und an den Texten arbeiteten - Emmanuel de Rougé, François Chabas, Charles Wycliffe Goodwin - ; ${ }^{10}$

- Heinrich Brugsch, geboren 1827, der Pionierdienste bei der Entzifferung des Demotischen leistete ${ }^{11}$ und die lexikalischen Erkenntnisse dieser Phase der Beschäftigung mit der ägyptischen Sprache in einem vielbändigen Wörterbuch einsammelte. ${ }^{12}$

Am Ende dieser Phase hatte man eine gewisse Routine im Umgang mit den Texten, man las sie jedoch mehr wortweise und weniger nach grammatischen Regeln. ${ }^{13}$

\section{Die Phase des Erschließens}

Um 1880 setzt in der Ägyptologie auf breiter Front eine Phase des systematischen Erschließens der Quellen ein, der Bodendenkmäler und der Texte. Ziel war jetzt die möglichst erschöpfende Erfassung und Auswertung des Materials. Selbstverständlich war es in Anbetracht der Menge des Erschließbaren schon damals eine Utopie, alles und jedes in beliebiger Detaillierung erfassen zu wollen. Es ging also de facto darum, in realistisch erreichbarer Detaillierung einen möglichst vollständigen Überblick zu gewinnen. Praktisch ging man so vor, dass man erstens die Aufgabe in Teilaufgaben zerlegte, die in mehr oder minder überschaubarer Zeit abzuschließen waren, und dass man zweitens eine Teilaufgabe nach der anderen zu erledigen trachtete, mit dem Ziel, mit der Summierung der Teilergebnisse ein Gesamtergebnis, den vollständigen Überblick, zu gewinnen (s. Abb. 3).

8 Durchbruch: CHAMPOLLION 1822, CHAMPOLLION 1824; Grammatik (posthum erschienen): CHAMPOLLION 1836-1841; Wörterbuch (posthum erschienen): CHAMPOLLION 1841-1843.

9 LEPSIUS 1837.

10 Überblick bei SCHENKEL 1996, 22-26.

11 BRUGSCH 1848.

12 BRUGSCH 1867-1882.

13 ERMAN 1929, 254-255. 


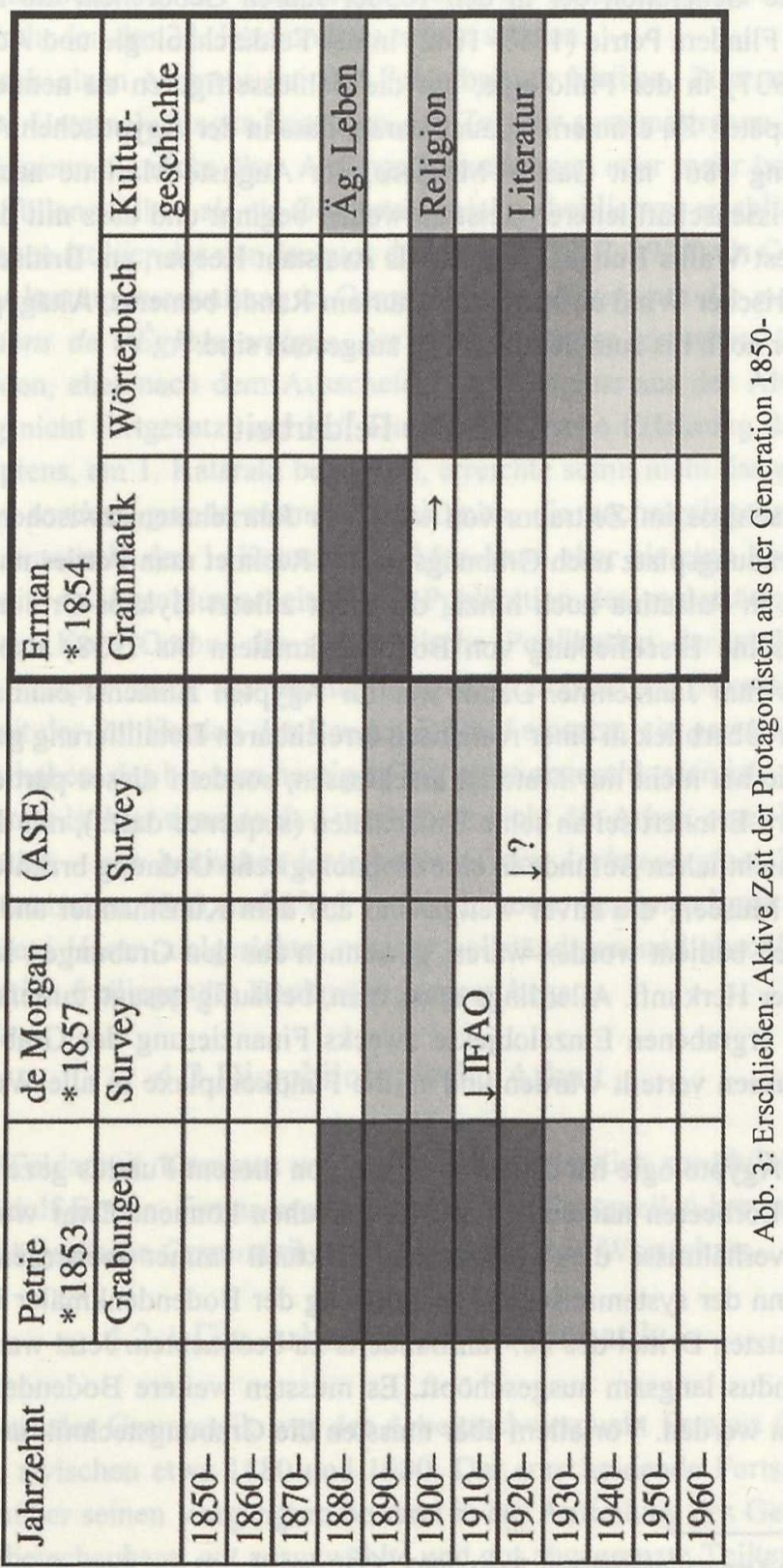


Es ist die Generation der in den 1850er Jahren Geborenen, die hier antrat, William Flinders Petrie (1853-1942) in der Feldarchäologie und Adolf Erman (1854-1937) in der Philologie, um die Schlüsselfiguren zu nennen. Andere Namen später. Zu erinnern ist auch daran, dass in der Ägyptischen Altertümerverwaltung 1861 mit Gaston Maspero, der Auguste Mariette nachfolgt, ein neuer, wissenschaftlicherer Geist zu wehen beginnt und dass mit dem Einzug von Ernest Wallis Budge, zunächst als Assistant Keeper, am Britischen Museum ein frischer Wind aufkam, dem, nur am Rande bemerkt, Altägyptenliebhaber leider noch bis zum heutigen Tag ausgesetzt sind. ${ }^{14}$

\subsection{Die Feldarbeit}

Petrie erschloss im Zeitraum von etwa vier Jahrzehnten, zwischen 1884 und 1926, Grabungsplatz nach Grabungsplatz. ${ }^{15}$ Rechnet man Petries nachfolgende Arbeiten in Palästina noch hinzu, die nicht zuletzt Hyksos-Orte erschlossen, dauerte seine Erschließung von Bodendenkmälern bis 1938, also insgesamt mehr als fünf Jahrzehnte. Damit war für Ägypten zunächst einmal ein vollständiger Überblick in einer realistisch erreichbaren Detaillierung gewonnen.

Petrie hat nicht nur Material erschlossen, sondern dieses partiell auch interpretiert. Erinnert sei an seine Staffeldaten (sequence dates), mit denen er die vorgeschichtlichen Befunde in eine chronologische Ordnung brachte. ${ }^{16}$

Die Museen, die zuvor weitgehend aus dem Kunsthandel und aus Raubgrabungen bedient worden waren, gewannen aus den Grabungen Material mit verbürgter Herkunft. Allerdings muss man, beiläufig gesagt, zutiefst bedauern, dass die ergrabenen Einzelobjekte zwecks Finanzierung der Grabungen über viele Museen verteilt wurden und so die Fundkomplexe in alle Welt zerstreut wurden.

Die Ägyptologie hat Jahrzehnte lang von diesem Fundus gezehrt und sich auf den Lorbeeren namentlich Petries ausruhen können. Zwar wurde, soweit die Zeitverhältnisse dies gestatteten, punktuell immer weitergearbeitet, ein Neubeginn der systematischen Erschließung der Bodendenkmäler ist erst wieder im letzten Drittel des 20. Jahrhunderts zu beobachten. Jetzt war der Petriesche Fundus langsam ausgeschöpft. Es mussten weitere Bodendenkmäler erschlossen werden. Vor allem aber mussten die Grabungstechnik und die Inter-

14 Zum Generationswechsel REID 2002, 172; dort S. 173 als Ende dieser Ära die Zeit etwa des 1 . Weltkriegs eingeschätzt.

15 PETRIE 1931, DROWER 1985.

16 Zur Bewertung aus heutiger Sicht KEMP 1982. 
pretationsmethoden des 19. Jahrhunderts durch die anspruchsvolleren Techniken und Methoden des 20. Jahrhunderts ersetzt werden.

Um noch einen Moment bei der Feldarbeit zu bleiben. Zum mindesten zwei andere Unternehmungen bezeugen den Zug zur systematischen ErschlieBung, auch wenn sie nicht über Anfänge hinauskamen oder mehr bestehende Lücken auffüllen wollten als ein Gesamtmaterial einheitlich zu erschließen.

Zu nennen ist hier der von Jacques de Morgan (1857-1924) als Generaldirektor der Altertümerverwaltung in Gang gebrachte Catalogue des monuments et inscriptions de l'Égypte antique, der in drei Bänden zwischen 1893 und 1895 erschien, aber nach dem Ausscheiden de Morgans aus der Altertümerverwaltung nicht fortgesetzt wurde. ${ }^{17}$ Die systematische Erfassung der Denkmäler Ägyptens, am 1. Katarakt begonnen, erreichte somit nicht das anvisierte Mittelmeer, sondern gerade einmal Kom Ombo, die nächstgelegene größere Fundstätte unterhalb des 1. Katarakts. - Man kann aber als eine Fortsetzung dieser Arbeit, als Anschlussarbeit an die Publikation des ptolemäerzeitlichen Tempels von Kom Ombo, die systematische Publikation der ptolemäischrömischen Tempel durch das Institut français d'archéologie orientale sehen, das 1897 mit der Publikation des Bandes Edfou I einsetzt, ein gewaltiges Publikationsvorhaben, das bis zum heutigen Tag nicht abgeschlossen ist.

$\mathrm{Zu}$ nennen ist hier dann auch - wenn auch nicht die Arbeit einer bestimmten Generation - ein britisches Unternehmen, der Archaeological Survey of Egypt, der zwischen 1893 und 1915 in 24 Memoirs, beginnend mit den Gräbern von Beni Hasan zielgerichtet mit der vollständigen und abschließenden Dokumentation freiliegender Denkmälergruppen begann.

\subsection{Die philologische Arbeit}

So viel zur Feldarbeit. Kommen wir schließlich und endlich zur Philologie und damit zu Adolf Erman. Erman erarbeitete von den Textquellen her von Grund auf neu die ägyptische Grammatik und den ägyptischen Wortschatz.

\subsubsection{Die Arbeit an der Grammatik}

Die Arbeit an der Grammatik war der Arbeitsschwerpunkt Ermans über zwei Jahrzehnte, zwischen etwa 1880 und 1900. Der entscheidende Fortschritt Ermans gegenüber seinen Vorgängern bestand in der Aufteilung des Gesamttextkorpus in überschaubare, gut ausgewählte und gut abgegrenzte Teiltextkorpora 
und die systematische Analyse dieser Teiltextkorpora. Die Ansätze zu dieser Arbeitsweise zeigen sich bereits bei seinen frühesten Arbeiten, denen des jungen Studenten bei der Beobachtung bestimmter Graphien beim Korrekturlesen des Glossars zur Edition des Papyrus Ebers $(1875)^{18}$, und denen des Doktoranden, bei der Rekonstruktion der Pluralbildung auf der Basis genauer Beobachtung der Graphien (1878). ${ }^{19}$ Es war dies dann, wie auch Erman selbst es sieht, ${ }^{20}$ das Erfolgsrezept bei der Erschließung des älteren Ägyptisch in den 1880er Jahren.

Grundlegende Bedeutung kommt vor allem zwei Werken zu, erstens der Neuaegyptischen Grammatik von 1880 und zweitens der Sprache des Papyrus Westcar von 1889, die sich nach dem Untertitel als „eine Vorarbeit zur Grammatik der älteren ägyptischen Sprache" versteht. In beiden Fällen wird aus der Fülle des überlieferten Textmaterials ein homogenes Teilmaterial ausgegrenzt, das differenziertere und konsistentere Aussagen über die Grammatik des Ägyptischen erbrachte als das zuvor praktizierte Einsammeln von Beobachtungen aus dem Gesamttextkorpus. In der Neuägyptischen Grammatik sind dies die Papyri aus dem Neuen Reich, in der Untersuchung zur älteren ägyptischen Sprache ein einzelner erzählender, inhaltlich relativ leicht verständlicher Text, die Erzählungen des Papyrus Westcar. Die Konzentration auf Teiltextkorpora zeigt, dass diese sich in grammatischer Hinsicht unterscheiden, konkret, dass das jüngere Ägyptisch der hieratischen Texte des Neuen Reiches eine andere Grammatik besitzt als das ältere Ägyptisch, etwa das Ägyptisch des Papyrus Westcar.

1889 bezeichnet den Höhepunkt der Ermanschen Grammatikarbeit. Hans Jacob Polotsky schwärmt von diesem Jahr geradezu als dem annus mirabilis der ägyptischen Grammatik. ${ }^{21}$ Denn nicht nur der Durchbruch zur ErschlieBung des älteren Ägyptisch wird mit der Arbeit am Papyrus Westcar geleistet, es datieren in dieses Jahr auch die so bedeutsame Entdeckung des Pseudopartizips als einer dem semitischen Perfekt entsprechenden Verbalform ${ }^{22}$ und die Beschreibung der für Polotsky - und die Dogmatisierung Polotskyscher Überlegungen in der sog. Standardtheorie - so bedeutsamen Markierungen von Verbalsätzen mit Partikeln wie ${ }^{c} h^{c} \cdot n \cdot{ }^{23}$ Erman selbst hat in seiner $\ddot{A} g y p t i s c h e n$ Grammatik von 1894 noch eine zusammenfassende Überblicksdarstellung der

18 ERMAN 1929, 257.

19 ERMAN 1878.

20 ERMAN 1929, $261 \mathrm{f}$.

21 Zuletzt POLOTSKY 1987-1990, 170. 261.

22 ERMAN 1889b.

23 ERMAN 1889a. 
Grammatik des älteren Ägyptisch vorgelegt, sich mit Spezialfragen wie z.B. der Frage des Verhältnisses des Ägyptischen zu den semitischen Sprachen (1892) beschäftigt und verbesserte Überblicksdarstellungen geliefert, so in einer zusammenfassenden Arbeit Zur Flexion des aegyptischen Verbums (1900, bereits auf dem Hintergrund der gleich zu nennenden Arbeit Kurt Sethes zu sehen) und bei der weiteren Überarbeitung seiner ägyptischen Grammatik, deren beste Auflage, die dritte, 1911 erschien. Erman wird jedoch seit etwa der Jahrhundertwende von seinen eigenen Schülern und Nacheiferern in der Grammatikarbeit überholt, namentlich von Kurt Sethe (1869-1934), mit dessen Mammutwerk über Das aegyptische Verbum, und zuletzt von Alan H. Gardiner, dessen Egyptian Grammar von 1927 dimensional und auch konzeptionell weit über alles hinausragt, was Erman je an zusammenfassenden Arbeiten zur ägyptischen Grammatik vorgelegt hat. Selbst die Neuaegyptische Grammatik, die Erman in Neubearbeitung noch einmal in 2. Auflage 1933 herausbrachte, ist kein Meilenstein geworden. Sie blieb nur deswegen Jahrzehnte lang die Standardgrammatik, weil sich andere Grammatiker nicht mit dem Neuägyptischen beschäftigten. Als eine neue Phase der Beschäftigung mit dem Neuägyptischen im letzten Drittel des 20. Jahrhunderts einsetzte, war Ermans Darstellung in wesentlichen Teilen, namentlich beim Verbum, rasch überholt. ${ }^{24}$

Erman interessierte sich für die späteste Stufe der ägyptischen Sprache, das Koptische. Was bei ihm aber gänzlich außen vor blieb, war das Demotische, und selbst das Hieroglyphisch-Ägyptische aus der Zeit nach dem Neuen Reich konnte ihn nicht sonderlich begeistern. Auf die Ausgrenzung des Demotischen wird später noch verschiedentlich die Sprache kommen.

\subsubsection{Die Arbeit am Wörterbuch}

Nachdem die ägyptische Grammatik im Laufe von zwei Jahrzehnten neu begründet war, verlagerte sich das Hauptinteresse Ermans auf die NeuerschlieBung des Wortschatzes direkt aus den Textquellen. Diese Arbeit nimmt ihn drei Jahrzehnte lang weitgehend in Beschlag, nämlich in den Jahren von 1897 bis, wenn man den Abschluss der Vorarbeiten zum Epochenjahr machen darf, 1926 oder, wenn das Erscheinen des letzten Hauptbandes des Wörterbuchs als Epochenjahr gelten soll, $1931 .{ }^{25}$ Auch diese Arbeit war typischerweise korpusbezogen, jetzt allerdings, anders als bei der teil-textkorpusbezogenen Gramma-

24 FRANDSEN 1974, ČERNÝ \& GROLl 1975, SATZINGER 1976; parallel die Belebung der gleich noch zu nennenden demotischen Grammatik: JOHNSON 1976.

ERMAN \& GRAPOW 1926-1963. 
tikarbeit auf das gesamte ägyptische, jedenfalls hieroglyphische und hieratische, Textkorpus. Wie bei der Grammatikarbeit wirft Erman Seitenblicke auf das späteste Ägyptisch, das Koptische, überspringt aber, und dies absichtsvoll, auch hier das Demotische. Im Aufruf zur Mitarbeit am Wörterbuch, der 1897 in der Zeitschrift für ägyptische Sprache und Altertumskunde erschien, heißt es:

„Das »Wörterbuch der ägyptischen Sprache« soll den gesammten [!] Sprachschatz umfassen, den die in hieroglyphischer (bez. hieratischer) Schrift geschriebenen Texte uns bewahrt haben; die demotischen und koptischen Texte sollen dagegen nur so weit herangezogen werden, als es die Erklärung hieroglyphisch geschriebener Worte verlangt.

Die Sammlung des Materiales erfolgt vermittelst des besonderen bei dem »Thesaurus linguae latinae« ausgebildeten Verfahrens, das es erlaubt, für jedes Wort sämmtliche [!] Belegstellen mit verhältnißmäßig geringer Mühe zu vereinigen. Bei der schließlichen Verarbeitung dieses Materiales werden dann natürlich nur die wesentlichsten dieser Belegstellen angeführt. “26

Womit Erman allerdings bei seinen Planungen nicht rechnete und nicht rechnen konnte, war die parallel einsetzende neue Datenflut, die sich aus der oben beschriebenen Feldarbeit - ich erinnere beispielsweise an die ptolemäischrömischen Tempel - und aus der systematischen Erschließung der angehäuften, aber unausgewerteten Museumsbestände - namentlich der Papyri - ergab. Das ursprüngliche Ziel einer vollständigen Erfassung des gesamten Sprachschatzes, erst recht sämtlicher Belegstellen, erwies sich als Utopie. Sie ist bis zum heutigen Tag nicht Realität geworden. (Sie wird dies aber hoffentlich noch, nachdem die Arbeit am Wortschatz an der Arbeitsstelle Altägyptisches Wörterbuch der Berlin-Brandenburgischen Akademie der Wissenschaften und in schwergewichtigen Kooperationsprojekten erneut in Angriff genommen worden ist.)

\subsubsection{Arbeiten zur Kulturgeschichte}

Der junge Erman war keineswegs von vornherein auf die Erforschung der ägyptischen Sprache aus. Noch als die Ausarbeitung einer Dissertation anstand, dachte er zunächst an ein historisches Thema. Es war Lepsius, der ihn jetzt in das linguistische Fahrwasser brachte. ${ }^{27}$ In seinen frühen Jahren blieb Ermans Horizont auch weiterhin ziemlich offen und verengte sich eigentlich

26 ERMAN 1897, auch ERMAN 1898a, englisch ERMAN 1898b.

27 ERMAN 1929, 259. 
erst mit der zunehmenden Bürde der Arbeit am Wörterbuch, die er selbst als eine Verzichtsleistung empfand. ${ }^{28}$

Sein Aegypten und aegyptisches Leben im Alterthum von 1885-87 basiert auf den damals bekannten Texten, vor allem aber auf einer systematischen Auswertung der Quellen, die in den großen Denkmälerwerken von Lepsius und auch Rosellini vorlagen. Diese umfassende Kulturgeschichte fällt noch in die Hauptphase der grammatischen Arbeiten Ermans. Es war aber auch, nebenbei bemerkt, dieses Aegyptische Leben, das seine ältesten Schüler anzog, nicht die Grammatik, in die er sie dann hineinzog. ${ }^{29}$ Zwei Jahrzehnte später, 1905, bereits in der Phase der Wörterbucharbeit, erschien seine Ägytische Religion, eine Arbeit die vielfach übersetzt wurde und die er selbst gegen Ende seines Lebens, 1934, unter dem Titel Die Religion der Ägypter noch einmal in Neubearbeitung vorlegte. Schließlich folgte weitere zwei Jahrzehnte später, 1923, jetzt im engen Umkreis der Arbeit an der Sprache angesiedelt, die Literatur der Ägypter, eine immer noch kulturgeschichtlich inspirierte Präsentation der wesentlichen Texte in Übersetzung.

Auch in diesem Bereich war die Initiative inzwischen teilweise an Ermans Schüler gegangen. Die Geschichte war von James Henry Breasted (18651935) übernommen worden (A History of Egypt, 1905; Quellenzusammenstellung Ancient Records of Egypt, 1906-1907), die Kunst von Heinrich Schäfer (1868-1957) (Von ägyptischer Kunst, 1919). Das Ägyptische Leben, das Erman immer noch als sein Werk sah, musste er aus Zeitmangel für die 1923 erschienene Neubearbeitung Hermann Ranke (1878-1953) überlassen, der daraus dann doch etwas anderes machte, als was der junge Erman hingeschrieben hatte: etwas weniger Schwungvolles, mehr Trocken-Wissenschaftliches. ${ }^{30}$

\subsubsection{Die Erfassung der Museumsbestände}

Nur am Rande und abschließend weise ich darauf hin, dass Erman als Direktor des Ägyptischen Museums dieselbe Erschließungsarbeit leistete, die er auf seinen Hauptarbeitsgebieten leistete: In seinem Ausführlichen Verzeichnis der aegyptischen Altertümer, Gipsabgüsse und Papyrus erfasste er zum ersten Mal, was anderen Museen bis zum heutigen Tag nicht gelungen ist, vollständig die ihm anvertrauten Museumsobjekte. Andere Unternehmungen, die seiner Initiative zu verdanken sind, die Veröffentlichung der Inschriften und Papyri, könnte man anfügen.

28 Ebd., 274, Anm. 1; 277.

29 SETHE \& SCHÄFER 1924.

30 ERMAN 1929, 278. 


\section{Erman und die anderen}

So viel zu Ermans Leistungen. Nun zu Ermans Einschätzung der Leistungen anderer.

\subsection{Das Urteil Ermans über seine Vorgänger}

Ermans Urteil über die ältere Ägyptologie kommt fast einer Verurteilung gleich: Er nimmt sie nur ausschnittsweise zur Kenntnis, und, soweit er sie zur Kenntnis nimmt, bringt er ihr nur teilweise eine gewisse Sympathie entgegen. Zunächst einmal ist klar, dass der junge Erman die ältere ägyptologische Literatur nicht ernsthaft studiert hat. Er bezieht aus der älteren Ägyptologie, sei es aus dem akademischen Unterricht, sei es aus sporadischer Lektüre ein gewisses Basiswissen. Ansonsten gewinnt er sein Wissen weitestgehend aus eigener Beschäftigung mit den originalen Quellen. Das hat zur Folge, dass an die Stelle des wenig durchstrukturierten, im Laufe von Jahrzehnten kumulierten Wissens strukturiertes Wissen in einigen zentralen Teilbereichen tritt. Es hat aber auch zur Folge, dass fallweise älteres Wissen unkontrolliert weiterverwandt wird, das ebenfalls von den Quellen her neu hätte erarbeitet werden müssen. Z.B. hat Erman das ägyptologische Transkriptionsalphabet in der von Brugsch verdorbenen Form übernommen, und dies, obwohl er Brugsch sonst schroff ablehnend gegenüberstand und obwohl er damit einen besseren Ansatz über den Haufen warf, den der von ihm eher geschätzte Lepsius aufgestellt hatte. ${ }^{31}$

\subsection{Erman und die Zeitgenossen}

Erman hat der altehrwürdigen und lebensvollen frankophonen Ägyptologie die kalte Schulter gezeigt, und das obwohl er von Hause aus in einer französischen Tradition stand, in Berlin nicht ohne Stolz das französische Gymnasium besuchte und, wie George Posener im Unterricht erzählte, sehr gut französisch sprach, besser als der gerade aus Paris nach Berlin angereiste Posener. Die Retourkutsche der Franzosen war die abwertend gemeinte Abstempelung der Kollegen „d'outre Rhin" als die „école de Berlin“, eine Bezeichnung, die mit der internationalen Anerkennung der „Berliner Schule“ allerdings dann doch zum Ehrentitel wurde.

Erman mied seine deutschen Zeitgenossen, so auch die bedeutenden Kollegen, den eine Generation älteren Heinrich Brugsch (1827-1894), dessen 
unstete, genialische Art ihm zuwider war, ${ }^{32}$ und den eine Generation jüngeren Wilhelm Spiegelberg (1870-1930), was beides zur Ausgrenzung des von diesen besetzten Spezialgebiets der Demotistik geführt haben dürfte, ${ }^{33}$ eines Gebietes, das sich Erman nie selbst erschloss und das folglich in der „Berliner Schule" keine zentrale Rolle spielte. Einzig Lepsius (1810-1884), inzwischen ein distinguierter und distanzierter alter Herr, scheint Eindruck auf Erman gemacht zu machen, sein Denkmäler-Werk, aber auch seine Persönlichkeit. Ihm verdankt er, wie oben bereits berichtet, den Anstoß zur Beschäftigung mit der Grammatik und nicht zuletzt ihm verdankt er auch seine Berliner Karriere. $^{34}$

Selbst ihm ergebene Schüler verstößt er, wenn sie ihm in den Weg kommen, so Heinrich Schäfer (1868-1957), der ihn, den langsam überforderten älteren Mann, als Direktor der ägyptischen.Abteilung der Staatlichen Museen ablöste. ${ }^{35}$ In der Autobiographie Mein Werden und mein Wirken fällt in diesem Zusammenhang, worauf Dietrich Wildung bei Gelegenheit der Tagung hinwies, nicht einmal der Name des Nachfolgers. Keine Spur der bahnbrechenden kunstgeschichtlichen Erkenntnisse Schäfers, die seit 1919 gedruckt vorlagen, in der letzten zusammenfassenden Kulturgeschichte Ägyptens aus der Feder Ermans, der Welt am Nil, von 1936!

\subsection{Erman und die zeitgenössische Wissenschaft}

Erman war, wie dies Antonio Loprieno in seinem Beitrag formuliert, kein Intellektueller oder, wie Erman selbst, der sich selbst gegenüber durchaus kritisch sein konnte, bekennt, ein „mittelmäßiger Mensch“ ${ }^{36}$ Was ihn auszeichnete war Bienenfleiß und ein gesunder Menschenverstand. Er trug Fakten zusammen und verstieg sich nicht.

Weder hat Erman zur zeitgenössischen Wissenschaft über Kärrnerdienste hinaus beigetragen, noch hat ihn die zeitgenössische Wissenschaft zu methodischer Reflexion angeregt. Er schwimmt mit auf der Welle des zeitgenössischen Positivismus. Bei seiner Beschäftigung mit der Sprache spielen Fakten aus Nachbarwissenschaften fallweise eine Rolle, so bei der Klärung der NisbaAdjektive anhand der arabischen Parallele, ${ }^{37}$ vor allem aber bei der Entde- 
ckung des Pseudopartizips als ein dem semitischen Perfekt verwandtes Paradigma ${ }^{38}$ und praktische Verfahrensweisen, so bei der Übernahme - auf Heinrich Schäfers (!) Hinweis ${ }^{39}$ - des Verzettelungsverfahrens des Thesaurus linguae latinae für das Projekt Ägyptisches Wörterbuch. Er ist aber bei all seiner Beschäftigung mit der Sprache praktisch unbeeinflusst von der zeitgenössischen Indogermanistik, d.h. von den Junggrammatikern, die seit etwa den 1870er Jahren die sprachwissenschaftliche Szene beherrschen. Ermans Ansatz ist nicht historisch-vergleichend.

Erman ist stolz auf die Menge des Faktenwissens, über das er verfügt und über das die Wissenschaft der Ägyptologie verfügt, aus der sich für das Fach ein Stellenwert im Kreis der anderen und vor allem der älteren historischphilologisch Nachbardisziplinen ergibt. Die kulturgeschichtliche Einordnung seiner Fakten liegt ihm dagegen weniger. Liest man sein Spätwerk, Die Welt am Nil von 1936, so hat man den Eindruck, dass sein Verhältnis zur pharaonischen Kultur trotz aller inzwischen angehäuften Einzelfakten immer noch das recht distanzierte ist, das seinen kulturgeschichtlichen Erstling, sein Ägyptisches Leben von 1885-87 kennzeichnet: Es ist ihm das meiste unsympathisch, unerfreulich, jedenfalls alles das, wozu ein Mensch seiner eigenen Zeit keinen unmittelbaren Zugang hat. Es missbehagt ihm der ägyptische literarische Stil, den er (1885) als „Phrasendrescherei“ abstempelt, die Religion, soweit sie Theologie ist und nicht Volksfrömmigkeit, die Kunst, soweit sie eine Ideologie zum Ausdruck bringt und nicht Alltagsszenen darstellt. Sein Urteil ist auf weiten Strecken unreflektiert.

\subsection{Zu Ermans Person}

Die Hauptursache für Ermans Einschätzung der Vorgänger und der Zeitgenossen liegt darin, dass er kein rechtes Gespür für die Würde anderer Menschen hatte. Er mochte nicht andere anerkennen, es sei denn, sie waren ihm ergeben. Der junge Erman hatte die Vorstellung, die Ägyptologie sei, wie man heute sagen würde, feuilletonistisch geworden und müsse aus dem Feuilletonismus wieder befreit werden. In seinen Erinnerungen spricht er abfällig von „Popularliteratur" und „Ägyptenschwärmerei““ ${ }^{40}$ Mit diesem Einwand freilich trifft er eigentlich nur seinen Lehrer Ebers, der in der Tat, dem Romanschreiben verfallen, keine Wissenschaft mehr betrieb. ${ }^{41}$ Es gab aber ebenso gut andere

Ebd., 263.

39 Ebd., 288.

40 Ebd., 255. 276.

41 Zu Ebers: FISCHER 1994. 
Personen in seiner näheren Umgebung, auf die dieser Vorwurf nicht zutraf. Z.B. und besonders Heinrich Brugsch, den er als Person nicht schätzte und den er als Wissenschaftler zu deklassieren suchte. ${ }^{42}$

Selbst ihm ergebene Schüler sahen diese Schwäche. Ich zitiere, etwas straffend, Hermann Grapow aus seinen Alterserinnerungen:

„[Friedrich Wilhelm Freiherr von] Bissing [war] von seiner Akademie als Vertreter Münchens in die Wörterbuchkommission (Berlin - Leipzig - Göttingen München) gewählt worden. Und so kam er alsbald etwa 1910 nach Berlin, um sich Erman in seiner neuen Würde vorzustellen und das Wörterbuch und seine Einrichtungen kennen zu lernen....

Bissing war schon am Tage zuvor gekommen, hatte aber Erman gerade im Weggehen gefunden, so daß eine Verabredung zu einer bestimmten Stunde für den nächsten Tag getroffen wurde. Als wir seine Schritte auf dem Gang hörten, machte Erman die schon nicht eben freundliche Bemerkung: Liebster, da kommt wohl unser Freund von gestern! Erman informierte Bissing sehr obenhin über das Wörterbuch und den Stand der Arbeit und sagte dann, ich zitiere wörtlich: ,Ach, Herr Grapow, bringen Sie uns doch einen Kasten mit der Präposition $m$ ', wozu bemerkt werden muß, daß wir im Laufe der Jahre schließlich über 90000 Belegstellen für diese häufigste ägyptische Präposition gesammelt haben, die damals noch in keiner Weise geordnet waren. Ich glaubte meinen Ohren nicht zu trauen, holte aber einen Kasten mit etwa 1600 Zetteln der Präposition und stellte ihn auf Ermans Tisch. Der sagte: ,Hier sehen Sie, Herr Kollege, einen kleinen Teil der Präposition $m$, die Sie ja wohl auch schon einige Male in den Texten gelesen haben werden!! Was tat Bissing? ... Er sagte: ,Ja, ja, gewiß, Herr Geheimrat. Das ist mir sehr interessant.' Als er dankend davon gegangen war, gab Erman mir den Kasten: ,So, Liebster, nun stellen Sie ihn man [berlinisch für ,mal'] wieder zurück. Unsern Freund aus München haben wir gehabt “ “ ${ }^{43}$

Voranstehendes auch zur Art der „Zusammenarbeit“ der federführenden Berliner mit den anderen deutschen Akademien im vom Reich finanzierten Wörterbuchprojekt.

Selbst durch die vornehm zurückhaltenden Formulierungen eines anderen ergebenen Schülers, Kurt Sethes, in einer Ansprache bei Gelegenheit des 70. Geburtstag Ermans am 31. Oktober 1924 verspürt man die distanzierte Haltung Ermans zu anderen als Wissenschaftler. Sethe spricht von den Wanderungen, die Erman gerne mit seinen Studenten unternahm:

„Wieder einige Jahre später finden wir den verehrten Jubilar mit zweien seiner Schüler im Harz auf dem Sonnenberg. Da werden die steilsten Schneisen des 
Odertales auf der einen Seite hinab-, auf der anderen wieder im Schweiße ihres Angesichts hinaufgeklettert, da werden Bäche in kunstvoller Weise verbaut und umgeleitet und andere Allotria getrieben. Freund Breasted, der damals bei Ihnen studierte und mit von jener Partie war, entwickelte da ungeahnte Fähigkeiten, die weit abseits der Wissenschaft und viel mehr auf dem Gebiet des Artistischen lagen. Mit Ihnen um die Wette sang er deutsche Studentenlieder und die famosen Sänge vom charcoalman und Michel Roy ..... Eine tiefinnere Fröhlichkeit erfüllte Sie damals, die Sie im Verkehr mit uns nie verließ. Sie erschien uns nicht nur in dem häuslichen Glück, das wir Sie genießen sahen, begründet, sondern als natürlicher Ausfluß der vorbildlichen Tüchtigkeit unseres Meisters, dem seine Leistungen ein beachtliches Kraft- und Sicherheitsgefühl gaben, das nicht verfehlte, uns einen tiefen, nachhaltigen Eindruck zu machen. Dieselbe Fröhlichkeit, die Sie den ganzen Tag singen und pfeifen ließ, (diesmal wars das Lied vom toten Schimmel, das Sie aus Schliersee mitbrachten) begleitete uns auch auf der nächsten Wanderung in Tirol, die Sie im Jahre 1895 mit dem inzwischen zum Privatdozenten erwachsenen Schüler unternahmen, damals, als wir es für unsere Berufspflicht hielten, die Pyramidenspitze im Kaisergebirge bei Kufstein bei afrikanischer Augusthitze zu besteigen, um uns hernach in Hinterbärenbad beim Ferlaner von den Strapazen dieser „Forschungsexpedition“ zu erholen. In diesem freundschaftlichen Verkehr mit Ihren Schülern, wie ihn diese Bilder aus der Vergangenheit wieder[sic]spiegeln, war das Fachgespräch, nicht selten zum Kummer der von ihrer Sache ganz erfüllten Schüler, streng verpönt, Sie wollten dann nur Mensch mit Menschen sein und haben Ihre Schüler damit in der Richtung erziehen helfen, daß bei ihnen das Menschliche nicht gar zu sehr hinter dem Wissenschaftlichen zurücktrete. Sie haben ihnen das Fachsimpeln, das der seiner Wissenschaft ganz hingegebene Student bekanntlich so ungern läßt, systematisch abgewöhnt. ““44

Sir Alan Gardiner - selbst das Beispiel eines Egozentrikers, als den er Erman darstellt, ein Mann, der die philologische Ägyptologie und die britische darüber hinaus als sein Territorium betrachtete - äußert sich in Aufzeichnungen, die eigentlich nur für seine Familie bestimmt waren, folgendermaßen:

"The Ermans were very hospitable, and particularly on Sundays liked to be joined by his pupils and ourselves in excursions to Wannsee or Grünewald [lies: Grunewald]. Or else we would be invited to their home in Steglitz - later it was Dahlem - where there would usually be their charming children, the elder girl Lotti and the young Anne-Marie (later Schaal) ${ }^{45}$ being particular favourites of mine. At these social gatherings there was never a word of Egyptology. I must confess that for the purposes of my private studies Erman was unsatisfactory. I have told how much I learned from reading over my Zettel [d.h. die für das Wörterbuch-

44 SETHE \& SCHÄFER 1924.

45 Vgl. zu Ermans jüngster Tochter Annemarie Schaal (1892-1992) die Ausführungen von Thomas Elsmann in diesem Band. 
Unternehmen bestimmten Zettel] with him. But if I had some minor discovery of my own on which I should have liked his opinion, he was definitely unhelpful. And so one of my early articles (on the title haty^y-^s [lies: h3ty-]) which was really excellent never got printed. If he did condescend to listen to some new point, he did so with the words: 'Aber machen Sie es schnell!' How different from Sethe, who would give one hours of his time, and was always of the greatest assistance to younger colleagues, even if he was at the same time violently combative. I am sorry to say that I could never regard Erman as a very fine character. He was definitely more cultured than most German professors whom I met, having been educated in a French school. He had also streaks of fairmindedness, as when he refused in the first World War to consent to any steps being taken to the disadvantage of former foreign assistants, like Breasted and myself. But in his work he was self-centred and unwilling to consult even so eminent a pupil or colleague as Sethe. Would "envy" be the right word to use of him, or is it too harsh? A favourite motto of his was:

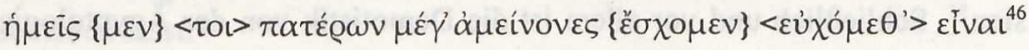
and another:

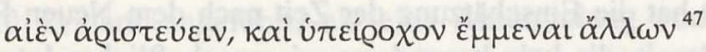

On the other hand, he was capable of self-blame if he found he had overlooked something obvious. I can hear him saying: 'Ich Esel!' Another saying of his from which I have at times derived comfort was: 'Ultra posse nemo obligatur.",48

Man wundert sich nach den zitierten Äußerungen nicht, dass, worauf Louise Gestermann in ihrem Beitrag hinweist ${ }^{49}$, in Ermans Briefwechsel die wissenschaftliche Diskussion weitgehend ausgespart bleibt.

46 Homer, Il. IV 405, nach dem Original emendiert, fehlerhafte Akzente stillschweigend verbessert; für die Bestätigung, dass es sich bei diesem und dem nachfolgend zitierten Vers nur um die angegebenen Stellen der Ilias handeln kann, danke ich Thomas A. Szlezák.

47 Homer, Il. VI 208 und XI 784, fehlerhafte Akzente stillschweigend verbessert. Homer, Il. VI 208-209 steht als Motto in Ermans Exlibris (vgl. ERMAN 1929, 177), das im Original vollständig so lautet :

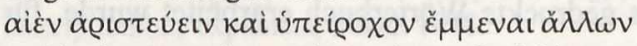

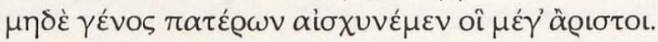

EX LIBRIS JOHANNIS PETRI ADOLFI ERMAN.

(zitiert aus meinem, vermutlich in den 1950er Jahren antiquarisch erworbenen, Exemplar der deutschen Ausgabe von Breasteds „Geschichte Ägyptens").

48 GARDINER 1986, 48-49 (die Kenntnis der biographischen Notizen verdanke ich Stephan J. Seidlmayer).

Vgl. S. 267. 


\section{Bilanz}

Wissenschaftsgeschichtlich gesehen, ist Ermans Überzeugung, „alles, aber auch alles" ${ }^{50}$ von den Quellen her neu erarbeiten zu müssen, ein Desaster. Was vor Erman liegt, ist den späteren Ägyptologengenerationen hinter dem Horizont verschwunden, als eine Insel gerade noch ausgenommen die weiter zurückliegende heroische Urzeit der Ägyptologie, die Geschichte der Entzifferung der Hieroglyphen. Da Erman tatsächlich nicht „,alles, aber auch alles“ von den Quellen her neu erarbeitet hat und auch nicht allein erarbeiten konnte, ist manches ungeprüft und ohne Quellenangabe weiter verwandt worden, was man hätte revidieren müssen, z.B. - wie oben gesagt - das ägyptologische Transkriptionsalphabet. Es ist auch manches dem Vergessen anheim gefallen, was weiterhin Wert gehabt hätte, z.B. nicht wenige Einzelbeobachtungen im Wörterbuch von Brugsch ${ }^{51}$ oder die ältere oder zeitgenössische Wortdiskus$\operatorname{sion}^{52}$. Schließlich und vor allem ist die Demotistik von der Ägyptologie teilweise abgekoppelt worden, ein Schaden, der bis zum heutigen Tag nicht voll behoben ist. Überhaupt hat die Einschätzung der Zeit nach dem Neuen Reich als einer reinen Verfallszeit, die kein besonderes wissenschaftliches Interesse verdient, noch lange nachgewirkt.

Andererseits und definitiv: Für uns Heutige beginnt die ägyptologische Philologie mit Erman. Wir alle stehen in der Tradition und in der Schuld der "Berliner Schule“.

Selbstverständlich sind die kulturgeschichtlichen „Nebenarbeiten“ Ermans im einzelnen überholt. Man kann die Bücher aber immer noch mit Gewinn lesen. Sehr viel falscher als die heute gängigen populärwissenschaftlichen Darstellungen sind sie auch nicht, und schlechter lesbar als diese sind sie gewiss nicht. Selbst die Arbeiten auf dem Gebiet der Grammatik, das sich inzwischen weit über Erman hinaus fortentwickelt hat, lohnen ein Wiederlesen. Manches, was Erman noch richtig sah, ist inzwischen wieder vergessen worden. Ich selbst bin z.B. bei der Transkription wieder in die Nähe von Erman zurückgekehrt und bin aktuell dabei, hier einen weiteren Schritt zurück zum frühen Erman zu wagen. ${ }^{53}$ Vor allem aber sind seine und seiner Mitarbeiter Arbeiten am Wörterbuch eine Fundgrube geblieben, so sehr, dass das Zettelarchiv, auf dessen Basis das gedruckte Wörterbuch erarbeitet wurde, für wert

50 So Erman in der Rückschau auf sein Aegypten und aegyptisches Leben im Alterthum in ERMAN 1929, 274.

51 Bemängelt z.B. von MEEKS 1977, 79.

52 Bemängelt bereits von GARDINER 1947, I, XX.

53 SCHENKEL 2005, 31-32. 
befunden wurde, ins Internet gestellt zu werden. ${ }^{54}$ Dieses Archiv ist nicht ersetzt und wird auch auf lange Sicht nicht leicht vollständig zu ersetzen sein. Und schließlich: Das gedruckte Wörterbuch der aegyptischen Sprache, herausgegeben von Erman \& Grapow ist bis zum heutigen Tag immer noch das ägyptische Wörterbuch.

Abgesehen von Fakten, die man bei Erman finden kann oder noch besser finden kann als anderswo, hat meines Erachtens Ermans Arbeitsweise nach wie vor ihren Stellenwert: die Konzentration auf Teiltextkorpora und die penible Beobachtung der graphematischen Oberfläche. Wenn ich persönlich werden darf: In diesem Fahrwasser schwimme ich mit meinen Untersuchungen zur Graphematik und zur Morphologie immer noch. ${ }^{55}$ Ich bin überzeugt davon, dass ich selbst mit dieser altmodischen Methode fallweise einen Beitrag zur Grammatik des Ägyptischen leisten konnte; vor allem aber davon, dass sich auf diesem Wege noch mancher Schatz heben lässt und sich nur auf diese Weise heben lässt.

Ich habe das digitalisierte Zettelarchiv als eine bleibende Hinterlassenschaft Ermans angefürt, als eine Ansammlung von Fakten, die weiterhin genutzt oder überhaupt zum ersten Mal voll genutzt werden kann. Aber auch in methodischer Hinsicht ist das Ermansche Wörterbuch-Projekt, die ErschlieBung des gesamten Wortschatzes mit der Gesamtheit der Belege aus dem Textkorpus immer noch zukunftsweisend. Der zu Ermans 150. Geburtstag realisierte Internet-Auftritt mit einer neuen Textdatenbank, dem Thesaurus linguae aegyptiae ${ }^{56}$ ist eine Realisierung des Ermanschen Grundkonzepts auf der Basis eines fortgeschrittenen Wissensstandes und fortgeschrittener Methoden mit den effizienteren Mitteln des Zeitalters von Computer und Internet.

\section{Literatur}

BREASTED 1905. Breasted, James Henry, A history of Egypt: from the earliest times to the Persian conquest, New York 1905.

BREASTED 1906-1907. Ders., Ancient records of Egypt: historical documents from the earliest times to the Persian conquest, Chicago 1906-1907.

BRUGSCH 1848. Brugsch, Heinrich, Scriptura Aegyptiorum demotica ex papyris et inscriptionibus explanata, Berlin 1848.

54 Konsultierbar im Thesaurus linguae aegyptiae, http://aaew.bbaw.de/tla/.

55 Spuren dieser Arbeit, über die zu berichten hier nicht der richtige Ort ist, und bibliographische Hinweise finden sich in SCHENKEL 2005. 
BRUGSCH 1867-1882. Ders., Hieroglyphisch-demotisches Wörterbuch: enthaltend in wissenschaftlicher Anordnung die gebräuchlichsten Wörter und Gruppen der Heiligen und der Volks-Sprache und Schrift der alten Ägypter, nebst deren Erklärung in französischer, deutscher und arabischer Sprache und Angabe ihrer Verwandtschaft mit den entsprechenden Wörtern des Koptischen und der semitischen Idio$\mathrm{me}=$ Dictionnaire hiéroglyphique et démotique: contenant en ordre méthodique les mots et les groupes les plus usités de la langue et de l'écriture sacrée et populaire des anciens Égyptiens, leur définition en français, en allemand et en arabe et l'indication de leur affinité avec les mots correspondants de la langue copte et des idiomes sémitiques, Leipzig 1867-1882.

CATAlOgUe DES MONUMENTS 1894-1909. Catalogue des Monuments et Inscriptions de l'Égypte antique, Wien 1894-1909.

ČERNÝ \& GROLL 1975. Černý, Jaroslav \& Sarah Israelit Groll, A Late Egyptian grammar, Rom 1975 (2. Aufl. 1978, 3. Aufl. 1984).

ChAmPollion 1822. Champollion, Jean-François, Lettre à M. Dacier ... relative à l'alphabet des hiéroglyphes phonétiques employés par les Égyptiens pour inscrire sur leurs monuments les titres, les noms et les surnoms des souverains grecs et romains, Paris 1822.

Champollion 1824. Ders., Précis du système hiéroglyphique des anciens Égyptiens: ou, recherches sur les éléments premiers de cette écriture sacrée, sur leurs diverses combinaisons, et sur les rapports de ce système avec les autres méthodes graphiques égyptiennes, Paris 1824.

Champollion 1835-1845. Ders., Monuments de l'Égypte et de la Nubie: planches / d'après les dessins exécutés sur les lieux, sous la direction de Champollion le Jeune, et les descriptions autographes qu'il en a rédigées, Paris 1835-1845.

ChAmpollion 1836-1841. Ders., Grammaire égyptienne: ou, principes généraux de l'écriture sacrée égyptienne appliquée à la représentation de la langue parlée, Paris 1836-1841.

Champollion 1841-1843. Ders., Dictionnaire égyptien en écriture hiéroglyphique, Paris $1841-1843$.

DESCRIPTION DE L'ÉGYPTE 1809-1826. Description de l'Égypte: ou, recueil des observations et des recherches qui ont été faites en Égypte pendant l'expédition de l'armée française, Paris 1809-1826.

DROWER 1985. Drower, Margaret S., Flinders Petrie: a life in archaeology, London 1985.

ERMAN 1878. Erman, Adolf, Die Pluralbildung des Aegyptischen: ein grammatischer Versuch, Leipzig 1878.

ERMAN 1880. Ders., Neuaegyptische Grammatik, Leipzig 1880 (2. Aufl. 1933).

ERMAN 1885-1887. Ders., Aegypten und aegyptisches Leben im Alterthum, Tübingen 1885-1887 (2. Aufl., neu bearb. von Hermann Ranke, 1923).

ERMAN 1889a. Ders., [ $\left.\zeta^{\prime} n\right]$ und die ihm analogen Formen, Zeitschrift für ägyptische Sprache und Alterthumskunde 27 (1889), 29-39.

ERMAN 1889b. Ders., Eine neue Art der aegyptischen Conjugation, Zeitschrift für ägyptische Sprache und Alterthumskunde 27 (1889), 65-84. 
ERMAN 1889c. Ders., Die Sprache des Papyrus Westcar: eine Vorarbeit zur Grammatik der älteren aegyptischen Sprache (Abhandlungen der Königlichen Gesellschaft der Wissenschaften zu Göttingen 36), Göttingen 1890 [sic].

ERMAN 1892. Ders., Das Verhältnis des Ägyptischen zu den semitischen Sprachen, Zeitschrift der Deutschen Morgenländischen Gesellschaft 46 (1892), 93-129.

ERMAN 1894. Ders., Ägyptische Grammatik: mit Schrifttafel, Litteratur, Lesestücken und Wörterverzeichnis, Berlin 1894 (2., gänzlich umgearb. Aufl. 1902, 3., völlig umgestaltete Aufl. 1911, 4., völlig umgestalt. Aufl. [mit geändertem Untertitel] 1928).

ERMAN 1905. Ders., Die ägyptische Religion, Berlin 1905 (2. Aufl. 1909).

ERMAN 1894. [Ders.], Ausführliches Verzeichnis der aegyptischen Altertümer, Gipsabgüsse und Papyrus, hrsg. von der Generalverwaltung, Königliche Museen zu Berlin, Berlin 1894.

ERMAN 1897. [Ders.], Aufruf / die akademische Kommission zur Herausgabe des Wörterbuches der ägyptischen Sprache: Ebers, Erman, Pietschmann, Steindorff, Zeitschrift für ägyptische Sprache und Altertumskunde 35 (1897), 111-112.

ERMAN 1898a.[ Ders.], Aufruf / die akademische Kommission zur Herausgabe des Wörterbuches der ägyptischen Sprache: Ebers, Erman, Pietschmann, Steindorff, Recueil de travaux relatifs à la philologie et à l'archéologie égyptiennes et assyriennes 20 (1898), 223-224.

ERMAN 1898B. [Ders.], A dictionary of the Egyptian language: an appeal to custodians and owners of inscriptions and papyri / The Academic Committee for the publication of the Dictionary of the Egyptian language, Proceedings of the Society of Biblical Archaeology 20 (1898), 167-168.

ERMAN 1900. Ders., Die Flexion des aegyptischen Verbums, Sitzungsberichte der Preußischen Akademie der Wissenschaften zu Berlin, Berlin 1900, 317-353.

ERMAN 1923. Ders., Die Literatur der Aegypter: Gedichte, Erzählungen und Lehrbücher aus dem 3. und 2. Jahrtausend v. Chr., Leipzig 1923.

ERMAN 1929. Ders., Mein Werden und mein Wirken: Erinnerungen eines alten Berliner Gelehrten, Berlin 1929.

ERMAN 1934. Ders., Die Religion der Ägypter: ihr Werden und Vergehen in vier Jahrtausenden, Berlin 1934.

ERMAN 1936. Ders., Die Welt am Nil: Bilder aus dem alten Ägypten, Leipzig 1936.

ERMAN \& GRAPOW 1926-1963. Ders. \& Hermann Grapow (Hg.), Wörterbuch der aegyptischen Sprache, Leipzig \& Berlin 1926-1963.

FISCHER 1994. Fischer, Hans, Der Ägyptologe Georg Ebers: eine Fallstudie zum Problem Wissenschaft und Öffentlichkeit im 19. Jahrhundert (Ägypten und Altes Testament 25), Wiesbaden 1994.

FRANDSEN 1974. Frandsen, Paul John, An outline of the Late Egyptian verbal system, Kopenhagen 1974.

GARDINER 1927. Gardiner, Alan H., Egyptian grammar: being an introduction to the study of hieroglyphs, Oxford 1927 (2. Aufl. London 1950, 3. Aufl. London 1957).

GARDINER 1947. Ders., Ancient Egyptian onomastica, Oxford 1947.

GARDINER 1986. Ders., My Early Years / Typed, photo-copied and bound by John Gardiner at Ballaghaue, Andreas, Isle of Man 1986. 
GlanVILLE 1947. Glanville, Stephen R. K., The growth and nature of egyptology: an inaugural lecture, Cambridge 1947.

GRAPOW 1973. Grapow, Hermann, Meine Begegnung mit einigen Ägyptologen, Berlin: Richard Seitz, 1973.

JoHnSON 1976. Johnson, Janet H., The Demotic verbal system, Chicago, Illinois 1976.

KeMP 1975. Kemp, Barry J, Dating Pharaonic cemeteries, Part I: non-mechanical approaches to seriation, Mitteilungen des Deutschen Archäologischen Instituts Abteilung Kairo 31 (1975), 259-291.

KEMP 1982. Ders., Automatic analyses of predynastic cemeteries: a new method for an old problem, The Journal of Egyptian Archaeology 68 (1982), 5-15.

KRAUSE 1988. Krause, Friedhilde, Richard Lepsius als Leiter der Königlichen Bibliothek zu Berlin, in: Elke Freier \& Walter F. Reineke (Hg.), Karl Richard Lepsius (1810-1884), Akten der Tagung anläßlich seines 100. Todestages, 10.-12.7.1984 in Halle, Berlin 1988, 60-72.

LEPSIUS 1837. Lepsius, Richard, Lettre à M. le professeur H. Rosellini ... sur l'alphabet hiéroglyphique, Rom 1937.

LEPSIUS 1849-1859. Ders., Denkmaeler aus Aegypten und Aethiopien: nach den Zeichnungen der von Seiner Majestät dem Könige von Preussen Friedrich Wilhelm IV. nach diesen Ländern gesendeten und in den Jahren 1842-1845 ausgeführten wissenschaftlichen Expedition, Berlin 1849-1859.

MeEKS 1977. Meeks, Dimitri, Notes de lexicographie (§ 5-8), Bulletin de l'Institut français d'archéologie orientale 77 (1977), 79-88.

PETRIE 1931. Petrie, William Flinders, Seventy years in archaeology, London 1931.

POLOTSKY 1987-1990. Polotsky, Hans Jakob, Grundlagen des koptischen Satzbaus. Atlanta, Georgia 1987-1990.

REID 2002. Reid, Donald M., Whose Pharaohs?: archaeology, museums, and Egyptian national identity from Napoleon to World War I. Berkeley, Los Angeles \& London 2002.

ROSELLINI 1832-1844. Rosellini, Ippolito, I monumenti dell'Egitto e della Nubia: disegnati dalla Spedizione Scientifico-Letteraria Toscana in Egitto / distribuiti in ordine di materie ... Pisa 1832-1844.

SATZINGER 1976. Satzinger, Helmut, Neuägyptische Studien. Die Partikel ir. Das Tempussystem (Beihefte zur Wiener Zeitschrift für die Kunde des Morgenlandes 6), Wien 1976.

SCHÄFER 1919. Schäfer, Heinrich, Von ägyptischer Kunst, besonders der Zeichenkunst: eine Einführung in die Betrachtung ägyptischer Kunstwerke, Leipzig 1919.

SCHENKEL 1988. Schenkel, Wolfgang, Erkundungen zur Reihenfolge der Zeichen im ägyptologischen Transkriptionsalphabet, Chronique d'Égypte 63 (1988), 5-35.

SCHENKEL 1996. Ders., Ägyptische Literatur und ägyptologische Forschung: Eine wissenschaftsgeschichtliche Einleitung, in: Antonio Loprieno (Hg.), Ancient Egyptian literature: history and forms (Probleme der Ägyptologie 10), Leiden, New York \& Köln, 21-38.

SCHENKEL 2005. Ders., Tübinger Einführung in die klassisch-ägyptische Sprache und Schrift, Tübingen 2005.

SEIDLMAYER 2004. Seidlmayer, Stephan Johannes, Der Thesaurus Linguae Aegyptiae im Internet, Göttinger Miszellen 203 (2004), 99-104. 
SETHE 1899-1902. Sethe, Kurt, Das aegyptische Verbum im Altaegyptischen, Neuaegyptischen und im Koptischen, Leipzig 1899-1902.

SETHE \& SCHÄFER 1924. Ders. \& Heinrich Schäfer, [Ansprachen Kurt Sethes und Heinrich Schäfers sowie Protokollierung weiterer Ansprachen und der Überreichung einer Nachbildung der Schreibergruppe Nr. 20001 des Berliner Museums, 1924] (das mir vorliegende Exemplar der hektographisch vervielfältigten Reinschrift stammt vermutlich aus dem Nachlass Adolf Ruschs, eines Schülers Ermans). 